\title{
Sebaceoma Arising from Nevus Sebaceous with Early Focal Carcinomatous Area
}

\author{
Joon Young Kim ${ }^{1}$, Ho Jik Yang ${ }^{1^{\star}}$, Joong Sun Lee ${ }^{2^{\star}}$, Hye Kyoung Lee ${ }^{3}$, Jong Hwan Kim ${ }^{1}$ \\ Departments of ${ }^{1}$ Plastic and Reconstructive Surgery, ${ }^{2}$ Dermatology, and ${ }^{3}$ Pathology, Eulji University Hospital, Daejeon, Korea
}

No potential conflict of interest relevant to this article was reported.

Nevus sebaceous (NS) is a hamartoma of the sebaceous gland that occurs congenitally and is characteristic of light-brown hairless plaque that mostly appears on the scalp and face. A widely known fact is that various neoplasms arise from NS. Secondary neoplasms arising from NS account for 5\%-6\%, most of which are benign. Malignancy is rather uncommon. In particular, sebaceous carcinoma was reported to be extremely rare worldwide [1]. The authors report a rare case of sebaceoma and sebaceous carcinoma occurring within a NS lesion and review the literature related to nevus sebaceous.

A 68-year-old man was admitted with a chief complaint of an occipital mass with rapid growth. Patient's history was significant for a yellow millet-shaped lesion in the left occipital region since birth. The patient also reported that he had injured the same spot at age 7 on a jagged stone and a scar had remained. Two years prior to the presentation, the patient struck the edge of a table with the same spot, which required unspecified treatment at a medical facility. The lesion began to grow rapidly for the past six months.

On examination, a hard yellow multinodular mass $(4 \times 6.5 \times 2.5$ $\mathrm{cm}^{3}$ ) was observed in the left posterolateral occipital scalp area

\section{Co-correspondence: Ho Jik Yang*}

Department of Plastic and Reconstructive Surgery, Eulji University Hospital, 95 Dunsanseo-ro, Seo-gu, Daejeon 35233, Korea

E-mail: drhjyang@eulji.ac.kr

Joong Sun Lee*

Department of Dermatology, Eulji University Hospital, 95 Dunsanseo-ro, Seo-gu, Daejeon 35233, Korea

E-mail: sun_lee@eulji.ac.kr

Received October 1, 2015 / Revised October 22, 2015 / Accepted November 29, 2015
(Fig. 1). The mass was accompanied by exudation and partial hemorrhage without any hair growth. The left cervical lymph node was palpable, but no other lymphadenopathy was identified. The magnetic resonance imaging study demonstrated the lesion to be a heterogeneously enhancing soft tissue mass with high signal intensity on T1-weighted image and medium signal intensity on T2-weighted image (Fig. 2). The mass did not involve the galea. An incisional biopsy suggested the lesion to be a skin adnexal tumor with sebaceous differentiation. Besides the suspected metastatic lymph in the left neck, the computed tomography (CT) study revealed no other abnormal findings in rest of the body. Ultrasound-guided fine-needle aspiration confirmed the left cervical lymphadenopathy to consist of benign nodules.

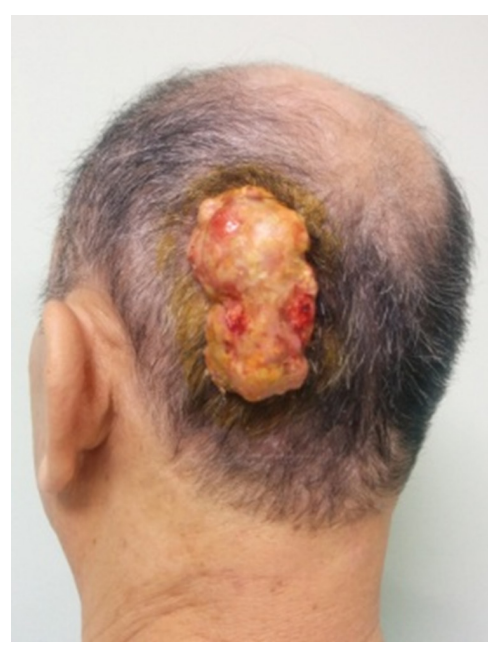

Fig. 1. Preoperative photograph of the scalp lesion. The $4 \times 6.5 \times 2.5 \mathrm{~cm}^{3}$ mass had grown rapidly in size over the past 6 months. 
With the tentative diagnosis, the patient underwent wide excision of the lesion with skin graft coverage. Under general anesthesia, the mass was excised with a 1-cm margin around the base. No gross invasion was observed, and intraoperative frozen section showed no malignant cells in the margins. The resulting scalp wound was covered with split-thickness skin graft.

Gross intraoperative examination revealed the mass to consist of multiple yellow nodules. Neither tumor necrosis nor hemorrhage were observed. Histologic examination showed most of the tumor to consist of sebaceoma with a focus of severe cytologic atypia consistent with carcinomatous change. On immunohistochemical examination, the sebaceoma was positive for epithelial membrane antigen (EMA), CK7, and MSH2 (Fig. 3). In the carcinomatous focus, p53 stained more intensely when compared to that of sebaceoma (Fig. 4). In addition, proliferation of undifferentiated cells was observed with a nevus sebaceous pattern in the basal part of skin epithelial cells (Fig. 5).

NS is a common congenital lesion. Benign tumors commonly arising from NS include trichoblastoma and syringocystadenoma papilliferum, while trichilemmoma and sebaceoma have also been reported. The malignant potential is extremely low, with less than $0.1 \%$ of NS harboring cancer cells. Among malignant cases, basal cell carcinoma is the most common, with sebaceous carcinoma being one of the rarest $[1,2]$.

In the present case, sebaceoma and sebaceous carcinoma accompanied by NS were confirmed based on biopsy results. When sebaceous carcinoma arises from NS, it can either occur alone or be accompanied by syringocystadenoma papilliferum, sebaceoma, or trichoblastoma [1,2]. Immunohistochemistry is essential in distinguishing between sebaceoma (EMA positive) and sebaceous carcinoma ( $\mathrm{p} 53$ positive).

Sebaceoma is a benign skin adnexal tumor with sebaceous differentiation, which can occur as a primary lesion or as a secondary one to either NS or seborrheic keratosis [3]. In this patient, the sebaceoma had developed secondarily to NS, which the patient presumably had since childhood. Muir-Torre syndrome (MTS) may be associated with sebaceous gland carcinomas that show sebaceous differentiation, including sebaceoma and sebaceous carcinoma. The syndrome demonstrates autosomal dominant in-

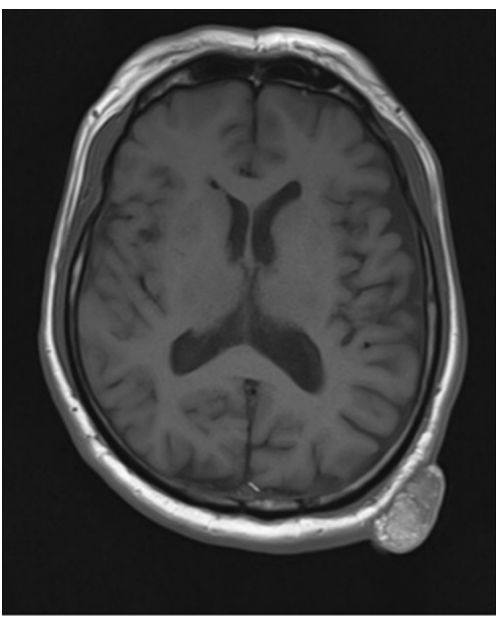

Fig. 2. Preoperative magnetic resonance imaging study demonstrates the lesion to be a large, lobulated, pedunculated, heterogeneous focal mass without galea aponeurotica involvement.

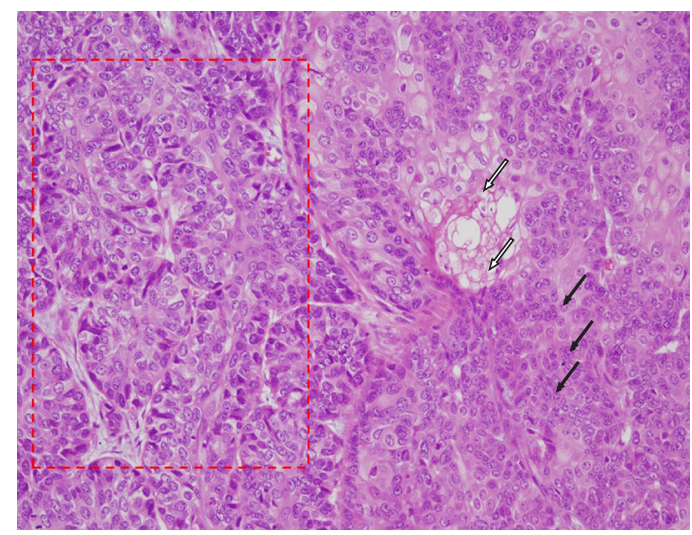

Fig. 3. Neoplastic cells composed of peripheral basaloid germinative cells (black arrows) and central vacuolated sebocytes (white arrows). Focal hyperchromatic carcinomatous area (red box) adjacent to the sebaceoma (H\&E, $\times 200)$.

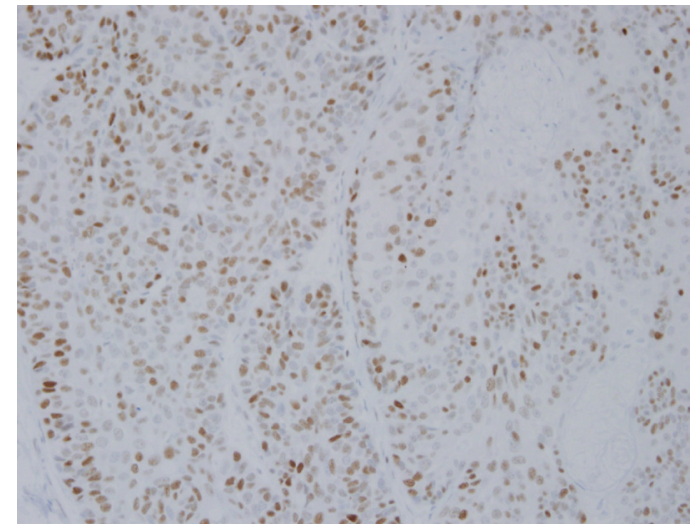

Fig. 4. Increased p53 positivity in the carcinomatous area (immunoperoxidase, $\times 200$ ). 


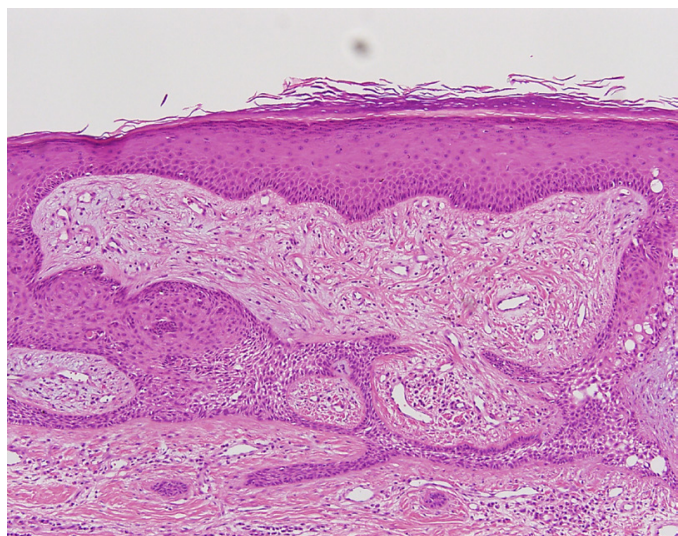

Fig. 5. Minute, benign-looking, abortive, basaloid cell proliferations suggesting nevus sebaceous in the overlying epidermis $(\mathrm{H} \& \mathrm{E}, \times 100)$.

heritance, and clinical presentation usually includes a visceral malignancy due to a mutation in MSH2, a mismatch repair protein $[3,4]$. However, our patient lacks the family history and medical history suggestive of MTS, and the immunohistochemistry of the lesion was positive for MSH2, indicating MTS to be unlikely.

Primary sebaceous carcinoma is a rare, aggressive malignant tumor that originates from the adnexal epithelium of the sebaceous gland and is associated with MTS. Local recurrence $30 \%-$ $40 \%)$ and distant metastases (20\%-25\%) are not rare [5]. Those sebaceous carcinomas arising from NS mainly occur on the scalp of women older than 40 years and is characterized by fast proliferation, which is suspected to be under the influence of sex hormones. Unlike primary sebaceous carcinoma, sebaceous carcino- mas of NS have no associations with MTS and no distant metastasis or recurrence have been reported. Overall, sebaceous carcinoma arising from NS are known to be of low-grade malignancy [1].

Various neoplasms can arise from NS, and visual examination does not always allow for proper differential diagnosis. Outgrowth of a mass from a pre-existing NS, as in the present case should raise the suspicion for the possibility of carcinoma, and the lesion should be differentiated among a list of neoplasms that accompanies sebaceoma. All diagnosis of sebaceous carcinoma warrants clinical workup for MTS, including history taking, clinical examination, dermatological examination, CT, upper gastrointestinal endoscopy, and colonoscopy.

\section{REFERENCES}

1. Izumi M, Tang X, Chiu CS, Nagai T, Matsubayashi J, Iwaya K, et al. Ten cases of sebaceous carcinoma arising in nevus sebaceus. J Dermatol 2008;35:704-11.

2. Cribier B, Scrivener Y, Grosshans E. Tumors arising in nevus sebaceus: a study of 596 cases. J Am Acad Dermatol 2000;42:263-8.

3. Dinneen AM, Mehregan DR. Sebaceous epithelioma: a review of twenty-one cases. J Am Acad Dermatol 1996;34:47-50.

4. Ponti G, Ponz de Leon M. Muir-Torre syndrome. Lancet Oncol 2005;6:980-7.

5. Nelson BR, Hamlet KR, Gillard M, Railan D, Johnson TM. Sebaceous carcinoma. J Am Acad Dermatol 1995;33:1-15. 